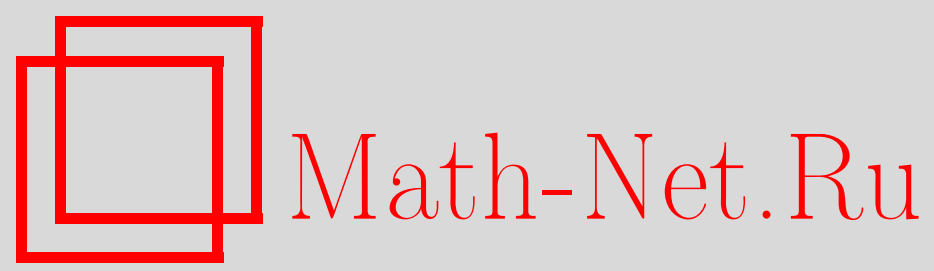

Ю. В. Хохрякова, K расчету эффективных упругопластических свойств многокомпонентных хаотически армированных композитов, Вестн. Сам. гос. техн. ун-та. Сер. Физ.-мат. науки, 2002, выпуск 16, 93-95

DOI: https://doi.org/10.14498/vsgtu104

Использование Общероссийского математического портала Math-Net.Ru подразумевает, что вы прочитали и согласны с пользовательским соглашением

http://www.mathnet.ru/rus/agreement

Параметры загрузки:

IP: 3.89 .197 .203

26 апреля 2023 г., 18:04:41 


\section{Ю.В. Хохрякова}

\section{К РАСЧЕТУ ЭФФЕКТИВНЫХ УПРУГОПЛАСТИЧЕСКИХ СВОЙСТВ МНОГОКОМПОНЕНТНЫХ ХАОТИЧЕСКИ АРМИРОВАННЫХ КОМПОЗИТОВ}

Найдены эффективные определяющие соотношения упругопластического деформирования многокомпонентных хаотически армированных композиционных материалов.

Целью настоящей работы является обобщения результатов работ [1,2]. Пусть рассматриваемый композиционный материал образован $n$ различными компонентами, соединенными между собой с идеальной адгезией, первый из которых играет роль матрицы - $V_{m}$; остальные $V_{f}$ - роль отдельных хаотически разориентированных включений, причем $V_{f}=\sum_{s=1}^{n} V_{s}$, где $V_{s}$ хаотически распределенные в матрице эллипсоидальные включения с главными полуосями $a_{1}^{(s)}, a_{2}^{(s)}, a_{3}^{(s)}$.

Исходные локальные уравнения упругопластического деформирования запишем в виде

$$
s_{i j}=2 \mu_{m ; f}\left(\sqrt{e_{k l} e_{k l}}\right) e_{i j}, \sigma_{p p}=3 K_{m ; f} \varepsilon_{p p},
$$

где $\mu_{f}=\sum_{s=1}^{n} \mu_{s}, K_{f}=\sum_{s=1}^{n} K_{s}$. Здесь $\mu_{m ; f}\left(\sqrt{e_{k l} e_{k l}}\right)$ - нелинейные модули пластичности сдвига; $K_{m ; f}$ - объемные модули; $s_{i j}=\sigma_{i j}-\frac{1}{3} \sigma_{k k}, \quad e_{i j}=\varepsilon_{i j}-\frac{1}{3} \varepsilon_{k k}$ - девиаторные части тензоров напряжений и деформаций; $\sigma_{i j}, \varepsilon_{i j}$ - тензоры напряжений и малых упругопластических деформаций.

Структуру композита будем описывать индикаторными функциями $\chi_{m}(\mathbf{r}), \chi_{f}(\mathbf{r}), \chi_{s}(\mathbf{r})$ $(s=\overline{1, n})$, равными единице в объемах $V_{m}, V_{f}, V_{s}$, и нулю - вне этих объемов соответственно. Здесь $\mathbf{r}=\left(x_{1}, x_{2}, x_{3}\right)$ - радиус-вектор координат.

Положение разориентированных в пространстве эллипсоидальных включений будем описывать характеристическими функциями $\chi_{s, a}(\mathbf{r}), a=\overline{1, s_{a}}$, равными единице в объемах $V_{s, \alpha}$ эллипсоидальных включений направления $\alpha$ конфигурации $s$.

Тогда

$$
\chi_{f}(\mathbf{r})=\sum_{s=1}^{n} \chi_{s}(\mathbf{r}), \chi_{s}(\mathbf{r})=\sum_{\alpha=1}^{s_{\alpha}} \chi_{s, \alpha}(\mathbf{r}), \mathrm{V}_{s}=\sum_{\alpha=1}^{s_{\alpha}} V_{s, \alpha} .
$$

Линеаризуем исходные уравнения (1), пренебрегая флуктуациями деформаций в пределах объемов матрицы и объемов эллипсоидальных включений конфигураций $s$, положив $\Lambda_{m ; f ; s}=\sqrt{\left\langle e_{k l}\right\rangle_{m ; f ; s}\left\langle e_{k l}\right\rangle_{m ; f ; s}}$. Здесь угловыми скобками обозначены средние значения по соответствующим объемам.

Тогда

$$
s_{i j}(\mathbf{r})=2\left(\mu_{m}+\sum_{s=1}^{n}\left[\mu_{s}\right] \chi_{s}(\mathbf{r})\right) e_{i j}(\mathbf{r}), \sigma_{p p}(\mathbf{r})=3\left(K_{m}+\sum_{s=2}^{n}\left[K_{s}\right] \chi_{s}(\mathbf{r})\right) \varepsilon_{p p}(\mathbf{r}) .
$$

Здесь $\left[\mu_{s}\right]=\mu_{s}-\mu_{m},\left[K_{s}\right]=K_{s}-K_{m}$.

Преобразуем систему уравнений, состоящую из уравнений (2), уравнений равновесия $\sigma_{i j, j}(\mathbf{r})=0$ и формул Коши $2 \varepsilon_{i j}(\mathbf{r})=u_{i, j}(\mathbf{r})+v_{i, j}(\mathbf{r})$, связывающих компоненты тензора деформаций с компонентами вектора скоростей перемещений, в систему уравнений в скоростях перемещений

$$
\mu_{m} v_{i, p p}^{\prime}+\left(K_{m}-\frac{1}{3} \mu_{m}\right)_{p, p i}^{\prime}-\tau_{i p, p}^{\prime}=0
$$




$$
\tau_{i j}(\mathbf{r})=-2 \sum_{s=1}^{n}\left[\mu_{s}\right] \chi_{s}(\mathbf{r}) e_{i j}(\mathbf{r})-\delta_{i j} \sum_{s=1}^{n}\left[\lambda_{s}\right] \chi_{s}(\mathbf{r}) \varepsilon_{p p}(\mathbf{r}), \lambda_{s}=K_{s}-\frac{2 \mu_{s}}{3} .
$$

С помощью тензора Грина эта система уравнений заменяется системой интегральных уравнений

$$
\varepsilon_{i j}^{\prime}(\mathbf{r})=\int_{V} G_{i k, l j}\left(\mathbf{r}-\mathbf{r}_{\mathbf{1}}\right) \tau_{k l}^{\prime}\left(\mathbf{r}_{\mathbf{1}}\right) d \mathbf{r}_{\mathbf{1}} .
$$

Осредним соотношение (2) по полному объему:

$$
\left\langle\sigma_{i j}\right\rangle=2 \mu_{m}\left\langle\varepsilon_{i j}\right\rangle+\delta_{i j} \lambda_{m}\left\langle\varepsilon_{p p}\right\rangle+2 \sum_{s=1}^{n}\left[\mu_{s}\right] c_{s}\left\langle\varepsilon_{i j}\right\rangle_{s}+\delta_{i j} \sum_{s=1}^{n}\left[\lambda_{s}\right] c_{s}\left\langle\varepsilon_{p p}\right\rangle_{s} .
$$

Здесь $c_{s}=\frac{V_{s}}{V}$. Из (5) видно, что для нахождения эффективных соотношений необходимо вычислить средние деформации $\left\langle\varepsilon_{i j}\right\rangle_{s}$. Для этого определим моменты

$$
\left\langle\chi_{s, \alpha}^{\prime} e_{i j}^{\prime}\right\rangle=\int_{V} G_{i k, l j}\left(\mathbf{r}_{1}\right)\left\langle\chi_{s, \alpha}^{\prime}(\mathbf{r}) \tau_{k l}^{\prime}\left(\mathbf{r}+\mathbf{r}_{1}\right)\right\rangle d \mathbf{r}_{1} .
$$

Воспользуемся тем, что функции $\chi_{s, a}(\mathbf{r})$ описывают только эллипсоидальные включения одного направления и будем считать, что корреляционные функции имеют вид

$$
\left\langle\chi_{s, \alpha}^{\prime}(\mathbf{r}) \tau_{k l}^{\prime}\left(\mathbf{r}+\mathbf{r}_{1}\right)\right\rangle=f_{k l}^{(s, \alpha)}\left(\frac{x_{1}^{2}}{\left[a_{1}^{s}\right]^{2}}+\frac{x_{2}^{2}}{\left[a_{2}^{s}\right]^{2}}+\frac{x_{3}^{2}}{\left[a_{3}^{s}\right]^{2}}\right) .
$$

Это допущение является обобщением гипотезы сильной изотропии на случай эллипсоидальной анизотропии в одном направлении. Тогда интегралы (6) вычисляются точно и их значения выражаются формулами

$$
\left\langle\chi_{s, \alpha}^{\prime} e_{i j}^{\prime}\right\rangle=\frac{c_{s, \alpha} Z_{i j k l}^{(s, \alpha)}}{2 \lambda_{m}}\left[\left\langle\tau_{k l}\right\rangle_{s, \alpha}-c_{f}\left\langle\tau_{k l}\right\rangle_{f}\right]
$$

Здесь $Z_{i j k l}^{(s, \alpha)}=S_{i j k l}^{(s, \alpha)}-\delta_{i j} \frac{v_{m}}{1+v_{m}} S_{p p k l}^{(s, \alpha)} ; S_{i j k l}^{(s, \alpha)}$ - компоненты тензора Эшелби, записанные в лабораторной системе координат эллипсоидальных включений направления $\alpha$.

Подставляя выражение (7) в известное соотношение [3], получим

$$
\left\langle\varepsilon_{i j}\right\rangle_{s, \alpha}=Q_{i j m r}^{(s, \alpha)} P_{m r k l}^{(s, \alpha)}\left[\sum_{q=1}^{n} c_{q}\left\langle\varepsilon_{k l}\right\rangle_{q}\right]+Q_{i j k l}^{(s, \alpha)}\left\langle\varepsilon_{k l}\right\rangle,
$$

где $P_{i j k l}^{(s, \alpha)}=\frac{1}{2 \mu_{m}}\left[2\left[\mu_{s}\right] Z_{i j k l}^{(s, \alpha)}+\delta_{k l}\left[\lambda_{s}\right] Z_{i j p p}^{(s, \alpha)}\right], \quad Q_{i j k l}^{(s, \alpha)}=\left[I_{i j k l}+P_{i j k l}^{(s, \alpha)}\right]^{-1}$.

Умножим (8) на $c_{s, \alpha}$ и, суммируя по всем направлениям $\alpha$, запишем уравнения для деформаций, осредненных по $V_{f}$ :

$$
\left\langle\varepsilon_{i j}\right\rangle_{f}=a_{i j k l}\left\langle\varepsilon_{k l}\right\rangle
$$

Здесь

$$
a_{i j k l}=c_{f}^{-1}\left[c_{m} I_{i j k l}+\sum_{s=1}^{n} K_{i j m r}^{(s)}\right]^{-1} \sum_{s=1}^{n} K_{m r k l}^{(s)}, K_{i j k l}^{(s)}=\sum_{\alpha=1}^{s_{\alpha}} c_{s, \alpha} Q_{i j k l}^{(s, \alpha)} .
$$

Подставляя (9) в (5), получим макроскопический закон упругопластического деформирования рассматриваемого композиционного материала:

$$
\left\langle\sigma_{i j}\right\rangle=E_{i j k l}^{*}\left(\Lambda_{m}, \Lambda_{1}, \ldots, \Lambda_{n}\right)\left\langle\varepsilon_{k l}\right\rangle,
$$

где $E_{i j k l}^{*}=2 \mu_{m} I_{i j k l}+\lambda_{m} \delta_{i j} \delta_{k l}+c_{f}\left[2\left[\mu_{f}\right] I_{i j k l}+\left[\lambda_{f}\right] \delta_{i j} \delta_{k l}\right] a_{i j k l}$ - эффективный тензор модулей пластичности. Поскольку в соотношение (10) входят величины $\Lambda_{m ; f ; s}$, то для расчета деформационных характеристик композита при конкретных способах нагружения его необходимо решать совместно с уравнениями (9). При этом следует задавать вид функций $\mu\left(\Lambda_{m ; f ; s}\right)$, который оп- 
ределяется на основе экспериментальных данных в соответствии с деформационными свойствами материалов компонентов.

Важным частным случаем общих соотношений (10) является модель композита, в котором эллипсоидальные включения ориентированы равновероятно. В этом случае

$c_{s, 1}=c_{s, 2}=\ldots=c_{s, s_{\alpha}}$. При этом тензоры четвертого ранга $K_{i j k l}^{(s)}=\sum_{\alpha=1}^{s_{\alpha}} c_{s, \alpha} Q_{i j k i}^{(s, \alpha)}$ будут изотропными и их общее представление будет иметь вид:

$$
K_{i j k l}^{(s)}=c_{s}\left(\alpha_{s} I_{i j k l}+\beta_{s} \delta_{i j} \delta_{k l}\right), \alpha_{s}=\frac{1}{15}\left[3 Q_{p q p q}^{(s)}-Q_{p p q q}^{(s)}\right] \beta_{s}=\frac{1}{15}\left[Q_{p q p q}^{(s)}-2 Q_{p p q q}^{(s)}\right] .
$$

Подставляя эти соотношения в тензорное уравнение (9) и выделяя объемную и девиаторную части, находим деформации, осредненные по объемам включений:

$$
\begin{gathered}
\left\langle e_{i j}\right\rangle_{s}=\frac{\alpha_{s}}{c_{m}+\sum_{s=1}^{n} c_{s} \alpha_{s}}\left\langle e_{i j}\right\rangle,\left\langle\varepsilon_{p p}\right\rangle_{s}=\frac{\gamma_{s}}{c_{m}+\sum_{s=1}^{n} c_{s} \gamma_{s}}\left\langle\varepsilon_{p p}\right\rangle,\left\langle e_{i j}\right\rangle_{f}=\frac{\sum_{s=1}^{n} c_{s} \alpha_{s}}{c_{f} c_{m}+c_{f} \sum_{s=1}^{n} c_{s} \alpha_{s}}\left\langle e_{i j}\right\rangle ; \\
\left\langle\varepsilon_{p p}\right\rangle_{f}=\frac{\sum_{s=1}^{n} c_{s} \gamma_{s}}{c_{f} c_{m}+c_{f} \sum_{s=1}^{n} c_{s} \gamma_{s}}\left\langle\varepsilon_{p p}\right\rangle, \gamma_{s}=\alpha_{s}-3 \beta_{s} .
\end{gathered}
$$

Тогда соотношения (10) принимают вид

$$
\begin{gathered}
\left\langle s_{i j}\right\rangle=2 \mu_{m}^{*}\left(\Lambda_{m}, \Lambda_{1}, \ldots, \Lambda_{n}\right)\left\langle e_{i j}\right\rangle,\left\langle\sigma_{p p}\right\rangle=3 K^{*}\left(\Lambda_{m}, \Lambda_{1}, \ldots, \Lambda_{n}\right)\left\langle\varepsilon_{p p}\right\rangle ; \\
\mu^{*}=\mu_{m}+\left[\mu_{f}\right] \frac{\sum_{s=1}^{n} c_{s} \alpha_{s}}{c_{m}+\sum_{s=1}^{n} c_{s} \alpha_{s}}, K^{*}=K_{m}+\left[K_{f}\right] \frac{\sum_{s=1}^{n} c_{s} \gamma_{s}}{c_{m}+\sum_{s=1}^{n} c_{s} \gamma_{s}} .
\end{gathered}
$$

Из (11) и правила смесей следует, что

$$
\Lambda_{s}=\frac{\alpha_{s}}{c_{m}+\sum_{s=1}^{n} c_{s} \alpha_{s}} e, \Lambda_{f}=\frac{\sum_{s=1}^{n} c_{s} \alpha_{s}}{c_{f} c_{m}+c_{f} \sum_{s=1}^{n} c_{s} \alpha_{s}} e, \Lambda_{m}=\frac{1}{c_{m}+\sum_{s=1}^{n} c_{s} \alpha_{s}} e
$$

Для решения этой системы необходимо задать вид функций $\mu\left(\Lambda_{m ; f ; s}\right)$, выбираемый на основе экспериментальных данных в соответствии с деформационными свойствами материалов компонентов. Решать систему (13) следует численными методами, вычисляя на каждом этапе итераций инварианты $\alpha_{s}, \beta_{s}$, полученные при обращении тензора $I_{i j k l}+P_{i j k l}^{(s)}$.

\section{БИБЛИОГРАФИЧЕСКИЙ СПИСОК}

1. Сараев Л. А., Хохрякова Ю. В. Упругопластические свойства трехкомпонентного хаотически армированного композиционного материала // Математическое моделирование и краевые задачи: Тр. одиннадцатой межвуз. конф. Ч.1. Самара: СамГТУ, 2001. С. 166-171.

2. Глущенков В. С., Сараев Л. А., Хохрякова Ю. В. Малые упругопластические деформации композиционнго материала, хаотически армированного эллипсоидальными включениями // Вестн. СамГУ. 2001. №2(20). С. 121125.

3. Сараев Л. А. Моделирование макроскопических свойств многокомпонентных композиционных материалов. Самара: СамГУ, 2000. 182c. 